\title{
Multimodal Technology for Municipal Inspections: An Evaluation Framework
}

\author{
Irina Kondratova ${ }^{1}$, Jeff Rankin ${ }^{2}$, and Ashley Goggin ${ }^{2}$ \\ ${ }^{1}$ NRC Institute for Information Technology, National Research Council Canada \\ 46 Dineen Drive, Fredericton NB E3B 9W4, Canada \\ Irina.Kondratova@nrc-cnrc.gc.ca \\ ${ }^{2}$ Department of Civil Engineering, \\ University of New Brunswick, Fredericton, Canada E3B 5A3 \\ \{rankin, i8r19\}@unb.ca
}

\begin{abstract}
Our paper discusses research on using multimodal interaction to improve usability of mobile data entry. This can benefit users of mobile technologies such as mobile workers, construction crews, and students collecting data during field trips. The evaluation of a mobile multimodal application for construction field work was conducted in the laboratory. Designing this laboratory experiment required a detailed definition of the type of device to be used, user requirements, including interaction techniques and usage context. During this work we discovered a need to develop a generic evaluation framework to assist in the selection of mobile technology and interaction techniques for further testing. The development of such a framework for municipal inspections with a focus on field inspections is discussed. This paper presents examples of using our framework to gain a better understanding of the contextual aspects influencing the feasibility of using mobile technology in the field.
\end{abstract}

Keywords: mobile technology, multimodal interaction, speech recognition, usability.

\section{Introduction}

Recent surveys of mobile Internet users show that usability is by far the biggest source of frustration among the users [1]. In particular, for learning applications, research shows that the most important constraining factors for widespread mobile learning (m-learning) adoption, along with battery life, are the screen size and user interface of most portable devices [2]. These usability problems are exacerbated when mobile devices are being used in the field for data collection and information management, where, in addition to above mentioned usability problems, users are constrained by the environmental conditions, including lighting, noise, weather, etc. This directly relates to the feasibility of using mobile devices for data collection by students during field trips.

Field trips play a vital role in engineering and science education, allowing students to acquire valuable knowledge and experience in the field while using field 
instruments and learning efficient data gathering techniques. Handheld devices have immense potential to be utilized during these field trips as data gathering devices, allowing the information collected in the field to be saved for future analysis in class [3]. Using handheld wireless devices in the field also enables real time communication with other groups of students as well as with a learning repository.

In order to assist users in managing mobile devices, user interface designers are starting to combine the traditional keyboard or pen input with "hands free" speech input [4], adding other modes of interaction such as speech-based interfaces that are capable of interpreting voice commands [5]. As a result, speech processing is becoming one of the key technologies for expanding the use of handheld devices by mobile users. In a recent eLearning technology foresight, technology-based education guru Tony Bates predicted that: "A new computer interface based on speech recognition will have a major impact on the design of e-learning courses" [6]. Currently, automated speech recognition (ASR) technology is being used in desktop e-learning applications for automated content-based video indexing for interactive elearning [7], audio-clip retrieval based on student questions [8], and, together with speech synthesis, to improve accessibility of e-learning materials for visually impaired learners [9].

Speech technology seems to be ideally suited for enhancing usability of m-learning applications designed for the mobile phone, where speech is a natural way of interaction and where a small screen size limits the potential for a meaningful visual display of information [10]. However, speech technology is limited to only one form of input and output - human voice. In contrast to this, voice input combined with the traditional keyboard-based or pen-based input model permits multimodal interaction in which the user has more than one means of accessing data in his or her device [11].

Multimodal interfaces allow speedier and more efficient communication with mobile devices, and accommodate different input modalities based on user preferences or on the usage context. For example, in a field trip scenario a civil engineering student would request information about the field structure (bridge, building, road, etc.) from the course repository using "hands free" voice input on a "smart phone" (hybrid phone-enabled PDA). The requested information would then be delivered as a text, picture, CAD drawing, or video, if needed, directly to the PDA screen. The user will be able to enter field notes in the forms using a portable keyboard or a pen, if appropriate, or via voice input during field data gathering. In addition to this, verbal field notes could be attached to the data collected in the form of an audio recording.

To evaluate a particular type of mobile technology for field work and choose appropriate modes of user interaction with a mobile device, technology evaluations are conducted in the field and in the laboratory. Laboratory evaluations of mobile technology use permits tightly controlled usability studies while simulating real-life usage environments [12]. To re-create field usage environments in the laboratory, indepth preliminary research should be conducted in order to single out a set of the most important parameters affecting technology use in the field. The success of laboratory testing often depends on the ability of a researcher to properly capture and recreate these contextual usage parameters. 


\section{Laboratory Evaluations for Mobile Field Data Entry}

In our ongoing research work on applications of mobile devices in the field for data collection and field notes taking, we developed a mobile application that allows a concrete inspector on the construction site to enter field testing data. Using this application, a concrete inspector can document the results of field concrete quality control tests using various interaction modes such as speech, stylus and a soft keyboard on the handheld device [13]. The application was evaluated in the laboratory by a group of users.

The evaluation was designed to recreate field conditions for concrete inspectors on the construction site. The typical construction site noise was recreated by conducting the study under noise conditions within a range of a typical construction site. The audio used to recreate the noise was recorded during concrete placing at a real construction site, thus providing a realistic evaluation environment for a mobile application that is designed to support data entry by concrete technicians. The experimental set-up insured a certain amount of mobility requiring a participant to travel from one table to another, a procedure that is similar to a concrete inspector performing quality control testing at the location of one concrete pour and then moving to another location on site [14].

To measure the awareness of participants of the surrounding environment during data entry, researchers projected random images on the walls of the lab and participants were asked to count certain types of images. A "safe" typical construction image depicted a building construction site with no heavy machinery present. A "danger" image projected was an image of a large concrete truck that field workers should be aware of while on site. During the experiment, participants were asked to count "danger" images shown while entering data or moving between data entry stations. As a result of this evaluation, we drew certain conclusions on the feasibility of using multimodal (speech and/or stylus and soft keyboard) data entry options in these conditions [15].

Designing this experimental evaluation in the laboratory required a significant amount of preliminary research on the type of device to be used, user requirements, including interaction techniques, usage context, etc. Therefore, through our experimental design and technology evaluation process we discovered a need for the development of the generic evaluation framework that will allow us to rapidly evaluate and select mobile technology and interaction techniques to be used for mobile data entry so that we can conduct follow-up laboratory and field testing.

\section{Mobile Municipal Technology Evaluation Framework}

The authors initiated the development of such a framework in a research project with the City of Fredericton (Canada) as a research client. The city's Information Technology Department wanted to conduct evaluations of several types of field municipal inspections in order to choose a mobile solution for a broad variety of inspections.

The project's primary deliverable was the development of a method for selecting various technology features for a given municipal inspection type. Six inspections 
from several of the municipality's departments served as the case studies in the development of the framework. The choice of inspections allowed representation of a broad range of possible contextual aspects and user requirements. Using this set of inspections as a case study, the aspects of an inspection that influence the selection of different forms of technology were determined, along with several conclusions on how mobile applications of technology may potentially improve an inspection process. Recommendations were developed for each individual inspection as well as an overall recommendation for a base mobile technology to be adopted by the municipality that would best fulfill the requirements of the majority of inspections.

\subsection{Methodology}

The mobile technology evaluation framework developed within this project can be used to evaluate mobile technology for a multitude of field applications. A block diagram of our framework is presented in Figure 1.

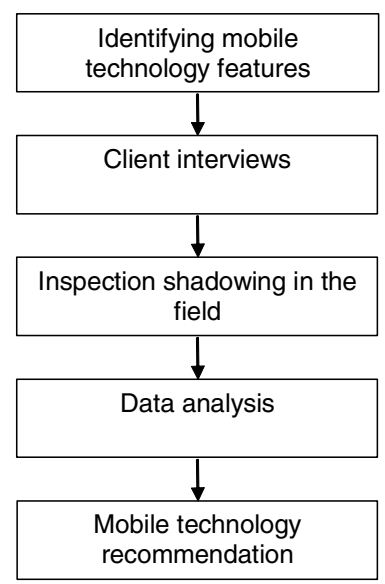

Fig. 1. Steps in the technology evaluation framework

Within the project we identified a number of technology attributes that should be taken into account when evaluating any mobile technology for field data entry applications. These attributes address specific requirements that clients may have as well as those factors derived from the field data gathering process, such as the information type and the surrounding environment.

The overall approach we have taken consisted of identifying certain perspectives of each individual inspection, data collection and analysis based on the templates we developed and recommendations on the technology solutions. Three perspectives of each individual inspection were defined as:

1. process steps for each individual inspection

2. information requirements

3. contextual aspects 
Process steps provided a breakdown of several distinct steps common among the majority of inspections to provide a comparative basis during analysis. The second perspective of information requirements detailed the type of information captured or accessed, which may range from textual to high resolution images. Several key issues affecting the selection of technology were identified within the third perspective, contextual aspects. For instance, environmental factors such as the weather would influence the feasibility of certain equipment types and may require a waterproofing feature, or excessive noise on the site might not permit a reliable speech data entry option for inspection data.

\subsection{Client Interviews}

Information for analysis was collected through a series of interviews with the client's primary contact as well as with the department supervisor for each of the respective inspections. Each participant was asked a set of pre-defined questions to gather such information as: their personal level of knowledge and experience with mobile and multimodal technology: which inspection they would like to have studied: identification of specific contextual aspects of the chosen inspection such as its location, duration, and any potential hazards that may be present: training or certification that may be needed for the inspector: and any possible improvements that they would like to make to the inspection process.

From the interviews it became evident that each participant had their own set of unique requirements and concerns to be addressed. Further, valuable data was gathered through a process of inspection shadowing to verify previously collected data and to gain further insight into all perspectives of a given inspection.

\subsection{Inspection Shadowing}

Although interviews with the department supervisors provided valuable information regarding the purpose of the inspection, further investigation was needed to discern the actual inspection process that takes place in the field. To accomplish this through an observational study, each inspector was shadowed during an actual inspection and was then asked a series of questions to determine their opinions of the inspection process and how various forms of mobile, and possibly multimodal, technology may be beneficial.

The questions we asked were designed to isolate specific environmental and contextual features of the inspection process that may impact the applicability of certain technology. The questions also assisted in determining what sort of tasks the inspector may be doing during the inspection. In addition to providing valuable information regarding the specific stages of several inspections, the shadowing process also provided a means of validating the participants' responses during the initial interviews. It was shown that an individual's responses regarding their own requirements and concerns may depend on their own specific responsibilities. Thus, by cross referencing data collected during the inspection shadowing process with 
information collected during client interviews, we were able to better define user requirements and usage context for each individual inspection.

As an outcome of this project, we developed a comprehensive procedure for analyzing technology requirements for a given city inspection, gathered detailed information in the field, and developed a systematic framework for analyzing the requirements of a given inspection [16].

\section{Application of the Framework}

To demonstrate an application of this analysis framework, the following section will illustrate the process we used to choose the appropriate mobile technology for a municipal concrete inspector and, as an example, for a group of civil engineering students on a field trip to the curb inspection site.

The following information on the concrete curb inspection was collected by the researcher via client interviews and inspection shadowing [16]. Concrete curb inspection of a concrete pour for curb and gutter is conducted to maintain standards in the product and the contractor's performance. It could be carried out under various weather conditions, including light rain. The potential risks at the site include traffic, excessive noise from equipment or vehicles, high speed traffic, large and heavy equipment and possible chemical exposure from curing compounds.

The inspector is moving around the worksite, using their hands which may get wet or dirty, using various pieces of equipment, speaking with others, making observations, and taking measurements. Currently all inspection information is collected using a standard paper form. No data privacy concerns were expressed by the participants. The current preferred method of communication is using a cell phone. Significant findings during inspection need to be reported immediately to a supervisor. Participants felt that usability and functionality will be the most important attributes of the device. A desire was expressed to have a permanent database of inspection results to aid in the review process for payment purposes and to examine the quality of the concrete at a later date. Participants felt that the device needs to be relatively simple to use and that the current inspection form should be reflected by the device.

Based on this information, the following mobile solutions for the concrete curb inspections were recommended to the city (a summary of this recommendation is presented in Table 1). We recommended to the city that the first choice for the concrete curb inspection would be a device similar to a Pocket PC (or a Smart Phone to provide a voice communication option) due to its small size and its ability to enter information efficiently with a stylus. The device needs to have a glare proof screen and waterproofing and should have a wireless connection to transmit significant findings to the office in real-time. It is likely that the interaction with the device will be limited to the use of stylus for the majority of the inspection due to the excessive noise at the worksite. Speech input may be feasible when certain equipment is not in use and there is minimal traffic activity. 
Table 1. Proposed mobile solution for a municipal concrete curb inspector

\begin{tabular}{|c|c|c|c|}
\hline \multicolumn{2}{|c|}{ Technology recommendation } & \multirow[t]{2}{*}{ Features required } & \multirow{2}{*}{$\begin{array}{ll}\text { Advantages } & \text { anc } \\
\text { disadvantages }\end{array}$} \\
\hline Preferred & Secondary & & \\
\hline Pocket PC & Tablet PC & $\begin{array}{l}\text { - Mobility } \\
\text { - Ease of storage } \\
\text { - Real-time connection for } \\
\text { automatic submission of forms } \\
\text { - Stylus for input } \\
\text { - Potential for speech entry } \\
\text { when machinery is not in } \\
\text { operation } \\
\text { - Rechargeable } \\
\text { - Glare proof coating } \\
\text { - Waterproof casing } \\
\text { - Potential for recording of } \\
\text { comments }\end{array}$ & $\begin{array}{l}\text { An advantage of } \\
\text { Pocket PC is that the } \\
\text { inspector may } \\
\text { remain mobile } \\
\text { throughout the } \\
\text { inspection. The } \\
\text { device is small } \\
\text { enough to be easily } \\
\text { stored, which allows } \\
\text { the inspector to use } \\
\text { their hands for other } \\
\text { tasks. }\end{array}$ \\
\hline
\end{tabular}

The advantages of the suggested technology are:

- Due to its small size, the device will allow the inspector to freely use hands and other equipment by being easily stored away in a pocket.

- The inspector will be able to move around the worksite without hindrance.

- The majority of the information collected on the current inspection form is checkbox, based on the selection of two or three pre-defined codes. Therefore, a device such as a Pocket PC with a stylus would be suitable for the inspection as the inspector would be able to select the appropriate code to convey the concrete curbing process' condition at each stage.

- A real time connection would allow for immediate submission of the completed inspection forms to the main office for review.

- A glare proof coating would allow the inspector to view the information in the field regardless of the lighting.

- Speech input may be advantageous as it would potentially allow the inspector to remain cognitive of the surroundings. However, speech would only be feasible as a data entry method when the heavy equipment is not in use and there is minimal traffic activity during certain sections of the inspection.

The main disadvantage in using a Pocket $\mathrm{PC}$ is its inability to record detailed written comments. Although the current inspection process does not require detailed comments to be recorded, comments would be an asset for the reviewers of the information to gain a better understanding of the condition of the concrete. However, a Pocket PC has an option of recording audio files with comments that could be attached to the inspection form.

The main advantage of the Tablet PC would be its ability to record written comments; a larger comments section may allow for a more complete description of the condition of the concrete. It is also important to note that the inspector must remain aware of the surroundings at all times due to the potential risks that are present. Therefore, a Tablet PC may also be beneficial in terms of a better awareness of the surroundings as the screen would allow displaying any images or information 
clearly for an inspector to see, and allow them to pay more attention to the surroundings at the same time.

Contrary to the Pocket PC usage recommended for a municipal inspector, a preferred mobile solution for a group of civil engineering students on a field trip to learn some basics of concrete testing would be a rugged Tablet PC with a glare proof coating. A Tablet PC will allow collecting data and additional notes in the field for a course report, while at the same time allowing more attention to be paid to the surrounding environment to provide for student safety on the construction site. Based on the assessment of the environmental conditions, the interaction technique with the device has to be limited to the use of a stylus due to the excessive noise at the worksite particularly when the concrete is being poured (noise level up to $95 \mathrm{~dB}$ ). However, speech input may be feasible when certain equipment is not in use and there is minimal traffic activity. Speech input may also be advantageous as it will allow the users to remain cognitive of their surroundings, as well as take additional field notes. The majority of the information collected on the current inspection form is a checkbox, based on the selection of two or three pre-defined codes, thus a stylus could be used efficiently to enter this information. Wireless connectivity will not be a requirement for this field work since all field notes could be downloaded to a desktop computer in class to produce a field report.

\section{Conclusions}

In many cases mobile technology for business, learning and field applications is underutilized due to the failure to choose an appropriate technology for particular usage conditions. The technology evaluation framework we developed in the research project with the local municipality as a research client, could serve as a generic framework for choosing mobile technology for field data collection applications, including m-learning applications.

The project's primary deliverable was the development of a method for selecting various features of technology for a given municipal inspection. Analysis was performed based on the information collected from the various interviews with the participants and from the process of shadowing the inspections. Both methods served as valuable tools for acquiring the necessary information. Although the two approaches complemented each other and served as a means of validating the information, the vast majority of the data was collected from the shadowing process in the field. While in the field, a broad range of contextual aspects for each inspection were identified through observing the inspector performing the actual tasks. This provided a better understanding of how a mobile device may potentially be used in the field. Information collected on each individual city inspection process will be utilized further in the development of the laboratory evaluations of mobile solutions for field work.

\section{Acknowledgments}

The researchers acknowledge the contributions provided by the employees at the City of Fredericton. This research project was supported by the Natural Science and Engineering Research Council of Canada and by National Research Council of Canada. 


\section{References}

1. Sadeh, N.: M-Commerce: Technology, Services, and Business Model. John Wiley \& Sons, Inc. New York (2002)

2. Trondsen, E.: Global Perspective on Mlearning. Macromedia Higher Education Leadership Forum San Francisco (2005)

3. Kravcik, M., Kaibel, A., Specht, M., Terrenghi, M.: Mobile Collector for Field Trips. Educational Technology \& Society 7(2), 25-33 (2004)

4. Wilson, L.: Look Ma Bell, No Hands! - Voicexml, X+V, and the Mobile Device. XML Journal (2004)

5. Sawhney, N., Schmandt, C.: Nomadic Radio: Speech and Audio Interaction for Contextual Messaging in Nomadic Environments. ACM Transactions on Computer-Human Interaction 7(3), 353-383 (2000)

6. Neal, L.: Predictions for 2002: E-Learning Visionaries Share Their Thoughts. eLearn Magazine. 2002 (1) (2002) 2

7. Zhang, D., Nunamaker, J.F.: A Natural Language Approach to Content-Based Video Indexing and Retrieval for Interactive E-Learning. IEEE transactions on multimedia. 6(3), 450-458 (2004)

8. Guenaga, M.L., Burger, D., Oliver, J.: Accessibility for E-Learning Environments. In: Miesenberger, K., Klaus, J., Zagler, W., Burger, D. (eds.) ICCHP 2004. LNCS, vol. 3118, pp. 157-163. Springer, Heidelberg (2004)

9. Jahankhani, H., Lynch, J.A., Stephenson, J.: The Current Legislation Covering E-Learning Provisions for the Visually Impaired. In: Shafazand, H., Tjoa, A.M. (eds.) EurAsia-ICT 2002. LNCS, vol. 2510, pp. 552-559. Springer, Heidelberg (2002)

10. De Freitas, S., Levene, M.: Evaluating the Development of Wearable Devices, Personal Data Assistants and the Use of Other Mobile Devices in Further and Higher Education Institutions. JISC Technology and Standards Watch Report: Wearable Technology (2002)

11. Jokinen, K., Raike, A.: Multimodality - Technology, Visions and Demands for the Future. In: Proceedings of the 1st Nordic Symposium on Multimodal Interfaces Copenhagen (2000)

12. Kjeldskov, J., Graham, C.: A Review of Mobile HCI Research Methods. In: Proceedings of the 5th International Symposium on Mobile Human-Computer Interaction (MobileHCI03) Udine, Italy (2003)

13. Kondratova, I.: Speech-Enabled Handheld Computing for Fieldwork. In: Proceedings of the International Conference on Computing in Civil Engineering ICCC 2005 Cancun, Mexico (2005)

14. Kondratova, I., Lumsden, J., Langton, N.: Multimodal Field Data Entry: Performance and Usability Issues. In: Proceedings of the Joint International Conference on Computing and Decision Making in Civil and Building Engineering Montréal, Québec, Canada (2006)

15. Lumsden, J., Kondratova, I., Langton, N.: Bringing a Construction Site into the Lab: A Context-Relevant Lab-Based Evaluation of a Multimodal Mobile Application. In: Proceedings of the 1st International Workshop on Multimodal and Pervasive Services (MAPS 2006) Lyon, France (2006)

16. Goggin, A.: Mobile Multimodal Technology for Inspections. University of New Brunswick. Fredericton (2006) 